\title{
Experimental Evaluation of Body Channel Response and Digital Modulation Schemes for Intra-body Communications
}

\author{
Jordi Agud Ruiz, Shigeru Shimamoto \\ Graduate School of Global Information and Telecommunication Studies, Waseda University \\ 29-7 building, 1-3-10 Nishi-Waseda, Shinjuku-ku, Tokyo 169-0051 Japan \\ E-mail: jordi.agud@toki.waseda.jp,shima@waseda.jp
}

\begin{abstract}
Intra-body communications is a short range communication solution suitable for Body Area Networks wherein the human body is used as the transmission medium. Currently, some studies have succeeded to transmit and receive simple information. However, intra-body communications could also provide broadband applications such as video streaming which will need high data rates. In this paper, firstly we investigate the propagation characteristics of the human body as a conductor of radio frequency signals. Afterwards, we evaluate five digital modulation schemes for intra-body communications. In the experiments, several distances between transmitter and receiver, symbol rates and frequency carriers are considered to investigate the optimal modulation scheme and the maximum transmission rate achievable. Constellation and eye diagrams and error vector magnitude are the performance parameters measured to evaluate the modulation accuracy.
\end{abstract}

Keywords-intra-body communications, body area networks, propagation characteristics human body, evaluation of modulation schemes for intra-body channels

\section{INTRODUCTION}

Body Area Networks (BAN) will allow new ways of wireless communications where the user will be able to interact with devices that move with the user or that are part of user's body [1]. One of the proposed technologies to achieve the goal of BAN is intra-body communication (IBC), a short-range communication solution between devices in which the human body is used as the signal transmission medium. We believe that IBC will allow not only the interaction between devices that move with the user body but also between users and the surrounding environment.

The number of potential applications using IBC is enormous. In [2], we presented several novel applications for passengers commuting to their workplace, schools, etc. For example, we proposed a new way of dynamic personalized advertising system by using IBC in trains. Passengers when touching a handle inside a train coach would be able to download advertisements in the most suitable format (audio, text or video) for the type of device that the passenger would be wearing. Moreover, we presented a service, called intrabody internet, whereby passengers could navigate internet, send/receive emails and even talk by means of their wearable devices (such as mobile phones, PDA and notebooks) not by using cellular networks but IBC. On the other hand, we also envisioned applications for handicapped people. For example, IBC could be used by blind people as a guidance system when walking in the streets, shopping centers and train stations. Whenever the blind person's shoes, that should have embedded IBC enabled sensors, step the IBC enabled ground line that should be installed to the surface of the sidewalk, communication could be carried out between both IBC enabled transceivers. The IBC enabled ground line could send information such as the current location or the destination and time departure of the closest track to his/her position in a train terminal. The data received by the IBC enabled foot sensor should be forwarded through the body to an IBC enabled earphone, so that the blind person could hear the information by voice. Moreover, e-payment could be another application suitable for blind people whereupon, for example, they would not need to purchase a train ticket in the vending machines. In this scenario, when the blind person was walking through the ticket gate, credit card information stored in his/her IBC enabled wristwatch could be transmitted to the IBC enabled foot sensor and then to the IBC enabled sensors located in the ground of the ticket gate that would be opened after confirmation of the credit card information. Deaf people could also benefit from IBC and, for example, receive the information that is transmitted through the speakers in train coaches or train stations by means of text information that could be displayed in an IBC enabled eye-glass display worn by the deaf person. As in the applications for blind people, the text information should be transmitted from an IBC enabled ground to the deaf person's IBC enabled foot sensor and then to the eye-glass display.

There are two main solutions for IBC: electric field type [3]-[5] and electromagnetic type [6] and [7]. By means of the former solution, data is sent across devices placed on or near the body by using a near field electric field. In the latter solution, the human body is treated as a waveguide with the radio frequency signals propagating through the body. Our research is based on the second solution. We aim to provide broadband IBC in order to satisfy future new applications (such as transferring multimedia files through the body) that will require high data rates and large bandwidths. In [8], we presented experimental results whereby we investigated the propagation characteristics of the human body as a conductor of radio frequency signals up to $1400 \mathrm{MHz}$ which were much 
higher than the maximum $100 \mathrm{MHz}$ frequency carrier considered in [7]. In this paper, we investigate the transmission characteristics of the human body considering higher frequency signals (up to $2.5 \mathrm{GHz}$ ), distances between transmitter (TX) and receiver (RX) up to $155 \mathrm{~cm}$ and test person sitting and standing. Moreover, we aim to establish a suitable frequency range for IBC based on the measurement results from the frequency domain response. On the other hand, we also aim to investigate the performance of five digital modulation schemes that are commonly used in $2 \mathrm{G}$ and $3 \mathrm{G}$ wireless systems. Related works, [3], [4] and [6], focused their studies mainly in the development of transceivers and also in the study of the propagation characteristics of the human body. These works employed mainly OOK, ASK and FSK as modulation schemes because of the easy implementation in the transceiver prototypes. In this paper, we evaluate several digital modulation schemes in order to determine the most suitable one for IBC. We measure constellation diagram, eye diagram and error vector magnitude (EVM) to evaluate the performance of each modulation scheme. We believe that an optimal modulation scheme for IBC would allow an increase of the low transmission rate achieved in the current related works based on the electromagnetic type solution (up to $9.6 \mathrm{Kbps}$ in [6]). The paper is organized as follows. Section II describes the experiment setup and the experiment results of the frequency domain response for the investigation of the body transmission characteristics. Afterwards, Section III presents first the experiment setup for the evaluation of digital modulation schemes applied to intra-body channels and then it shows the measurement results. Finally, Section IV contains the conclusions of this paper.

\section{ANALYSIS OF THE TRANSMISSION CHARACTERISTICS OF THE INTRA-BODY PROPAGATION CHANNNEL}

\section{A. Experiment Setup}

The experiments were performed in a classroom with only desks and chairs. The frequency domain response of the intrabody propagation channel was measured using a network analyzer. Fig. 1 shows the measurement scenario where radio signals were generated in the network analyzer, input in the human body through the TX, received in the RX and finally evaluated in the network analyzer. The input signal power was selected based on [9] in which it is investigated the effects of electromagnetic fields up to $300 \mathrm{GHz}$ and it is recommended a basic limit exposure of $0.08 \mathrm{~W} / \mathrm{kg}$ in order to be safe for the human body. We employed an input power of just $15 \mathrm{dBm}$ that is much lower than the $37 \mathrm{dBm}$ maximum signal power that could be transmitted in case of considering an average body weight of $65 \mathrm{~kg}$. TX and RX were configured by a $2 \mathrm{~cm}$ diameter copper signal electrode touching the skin and a $10 \mathrm{~cm}$ by $5 \mathrm{~cm}$ ground (GND) circuit board distant $1.4 \mathrm{~cm}$ from the signal electrode. This configuration is based on the analysis carried out in [8] and later investigations considering several signal and GND electrode diameters (from $1 \mathrm{~cm}$ to $3 \mathrm{~cm}$ ), GND circuit board sizes $(10 \mathrm{~cm}$ by $5 \mathrm{~cm}, 5 \mathrm{~cm}$ by $5 \mathrm{~cm}$ and $2 \mathrm{~cm}$ by $2 \mathrm{~cm}$ ), distances between the signal electrode and the circuit board (from $0.7 \mathrm{~cm}$ to $2.1 \mathrm{~cm}$ ) and considering TX and/or RX with or without GND electrode touching the skin. Note that the GND of both TX and RX are connected to the electrical ground

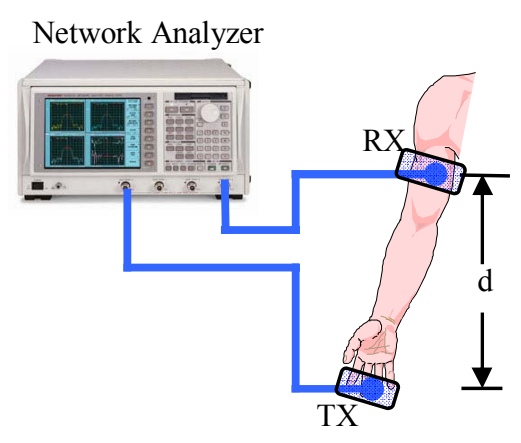

Figure 1. Experiment scenario for the analysis of the frequency domain response.

of the network analyzer.

Fig. 2 illustrates the measurement models employed in the experiments of this and next sections: arm to arm model, foot to head model and foot to arm model. In the arm-arm model, the TX position was always fixed on one hand and the RX was located along both arms (distances from $20 \mathrm{~cm}$, i.e. hand to wrist of the same arm, up to $160 \mathrm{~cm}$, i.e. hand of one arm to wrist of the other arm, were considered). The foot-head model was defined based on the idea of guidance application for blind people explained previously. The test person's foot stood on the TX and several RX measurement positions were settled (distances of $90 \mathrm{~cm}$ and $155 \mathrm{~cm}$ that corresponded to a distance between the foot and, the waist and the ear respectively). In the foot-arm model, the TX was located in the foot and the RX in the wrist (the distance between TX and RX was approximately $180 \mathrm{~cm}$, the maximum considered in this paper). This measurement model was defined based on the idea of the epayment application for handicapped people.

\section{B. Experiment Results and Discussions}

Figs. 3 and 4 illustrate the frequency domain responses obtained for both arm-arm and foot-head measurement models respectively. The horizontal axis represents the frequency in $\mathrm{MHz}$ (from $1 \mathrm{MHz}$ to $2.5 \mathrm{GHz}$ ) and the vertical axis the power of the received signal in $\mathrm{dBm}$. We measured the received signal power considering the test person in both sitting and standing test condition. Both graphs show that the attenuation of the received signal increases when the frequency also increases. Moreover, the decay of the received signal power is faster when the distance between TX and RX is longer. We also observe from both figures that sitting and standing test conditions yield similar results for all the distances and,

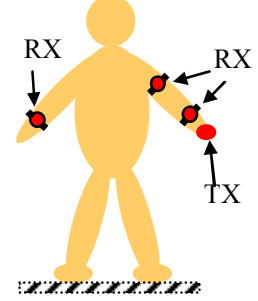

(a)

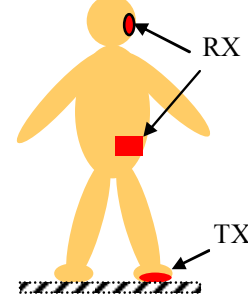

(b)

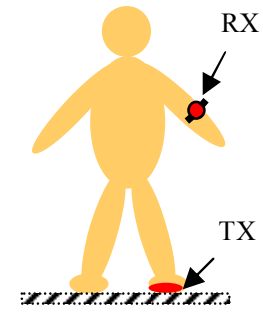

(c)
Figure 2. Measurement models: (a) arm-arm model, (b) foot-head model, (c) foot-arm model. 


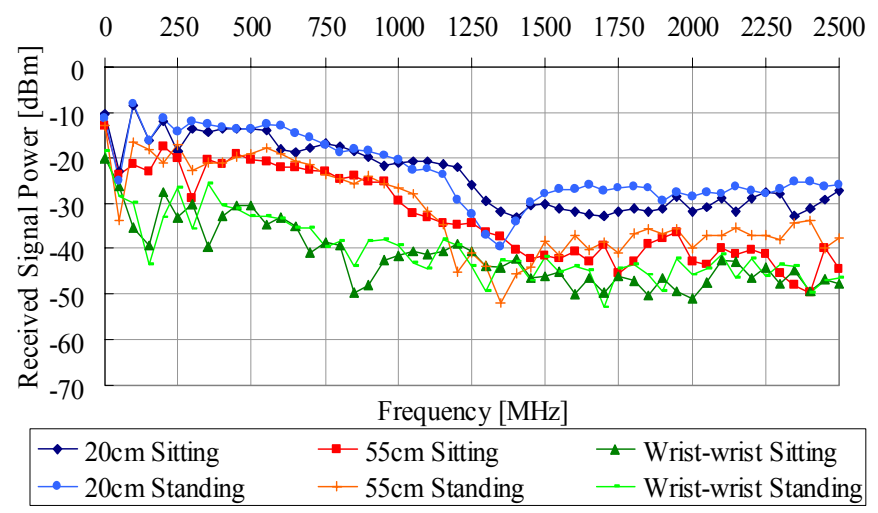

Figure 3. Average received signal power of arm-arm model.

$\begin{array}{lllllllllll}0 & 250 & 500 & 750 & 1000 & 1250 & 1500 & 1750 & 2000 & 2250 & 2500\end{array}$

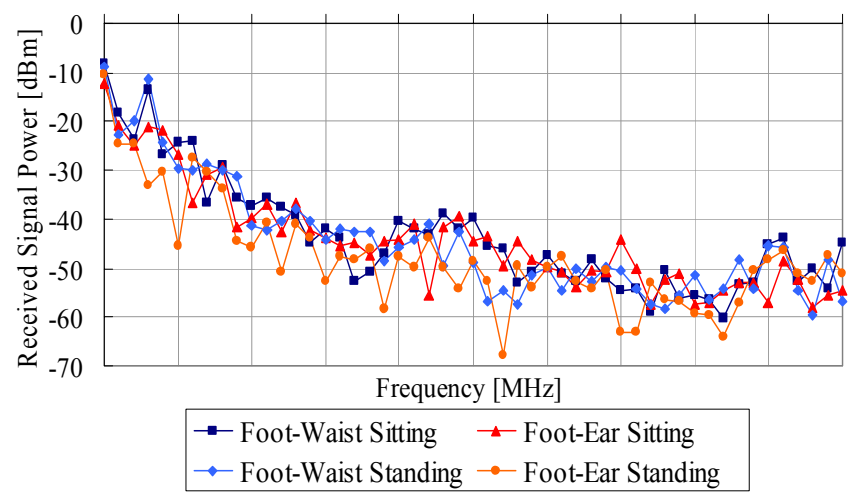

Figure 4. Average received signal power of foot-head model.

therefore, we can affirm that there is not a better position for this kind of communication. On the other hand, we believe that a suitable frequency range for IBC could be up to $600 \mathrm{MHz}$, based on the zone with the lowest attenuation results from both figures.

\section{Performance Evaluation of Digital Modulation SCHEMES FOR INTRA-BODY CHANNELS}

\section{A. Experiment Setup}

The measurements were performed within the same environment conditions as the former experiment. Fig. 5 shows the measurement scenario wherein a signal generator generates and modulates a radio signal that is transmitted by the TX through the human body. The received signal is detected, demodulated and evaluated in a wireless communication analyzer. MSK, BPSK, QPSK, 8PSK and 16QAM were the five modulation schemes analyzed in the measurements. The frequency carriers employed were $200 \mathrm{MHz}$ and $600 \mathrm{MHz}$, based on the suitable frequency range determined previously. Moreover, symbol rates from $100 \mathrm{Ksps}$ to $5 \mathrm{Msps}$ were employed in the experiments in order to analyze the maximum data rates achievable through each modulation scheme. The TX and RX configuration was the same as in the former experiment.

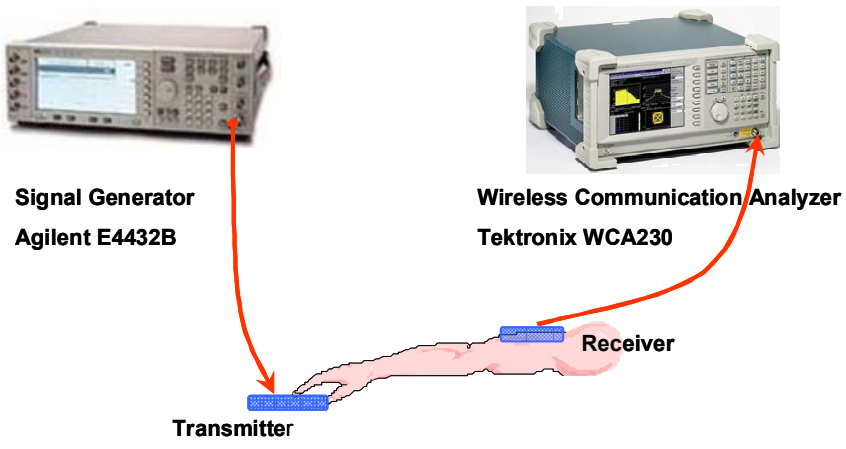

Figure 5. Experiment scenario for the evaluation of modulation schemes.

\section{B. Experiment Results and Discussions}

The constellation diagram, eye diagram and EVM are measured to evaluate the performance of MSK, BPSK, QPSK, 8PSK and 16QAM, considering several symbol rates and distances between TX and RX. All the results of this paper show the performance of the modulation schemes when the frequency carrier is $200 \mathrm{MHz}$. Note that similar results were obtained in case of a frequency carrier of $600 \mathrm{MHz}$.

\section{1) Constellation Diagram}

On the one hand, Fig. 6 presents the results when symbol rate (SR) is $100 \mathrm{Ksps}$ and the distance between TX and RX (DTR) is $60 \mathrm{~cm}$. Both results from intra-body channel and air propagation channel (i.e. TX and RX without touching the human body) are shown to compare the performance of both transmission mediums within the same experimental conditions. The results show that the intra-body propagation channel yields better performance in all modulation schemes than the air propagation channel which is more affected by distortion and external noises. In other words, the body acts as a kind of wire and, therefore, the intra-body propagation channel is not as affected by external interferences as the air propagation channel. Focusing on the results of the intra-body propagation channel, Fig. 6k shows that MSK provides the best performance as the received symbols are very close to the reference constellation points. The worst results are provided by 16QAM (see Fig. 6n) with received points far from the constellation points and 8PSK with received signals dispersed all along the diagram. BPSK and QPSK perform well with most of the received symbols quite close to the reference points. On the other hand, Figs. 6c, 6f, 6i, 61 and 60 show the results of analyzing the effects of increasing the distance between TX and RX (from $60 \mathrm{~cm}$ to $155 \mathrm{~cm}$ ) and also the symbol rate (from $100 \mathrm{Ksps}$ to $2.5 \mathrm{Msps}$ ) in the intra-body propagation channel. The increase of transmission rate and distance causes that the distortion also increases in all modulation schemes. BPSK, QPSK and MSK provide good performance with received points close to the decision points. 16QAM yields better results than in Fig. 6n. 8PSK is the worst case (see Fig. 6i) with received symbols dispersed along all diagram and, therefore, without received points close to the reference points.

Although it is not shown in this paper, the increase of the symbol rate and the distance between TX and RX to the 


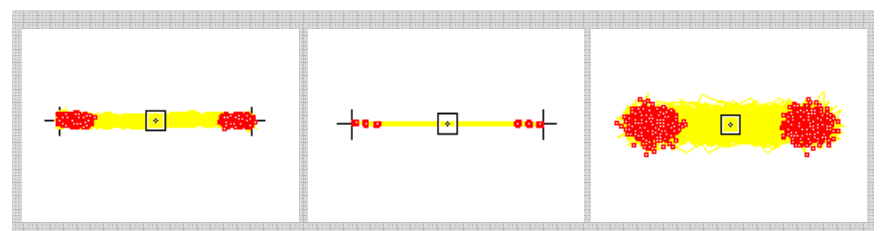

(a)

(b)

(c)

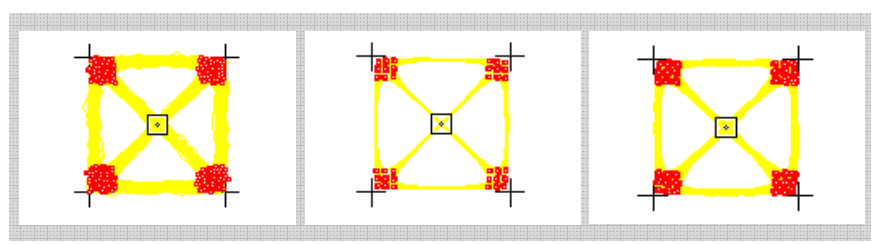

(d)

(e)

(f)

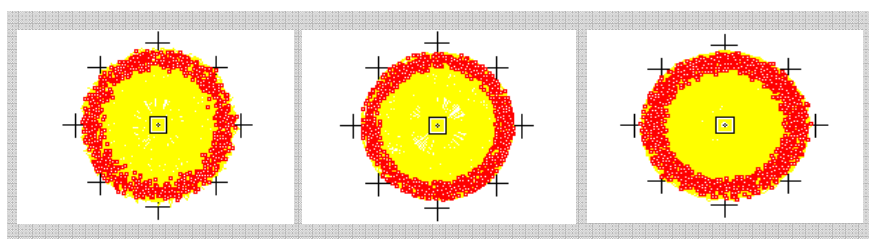

(g)

(h)

(i)

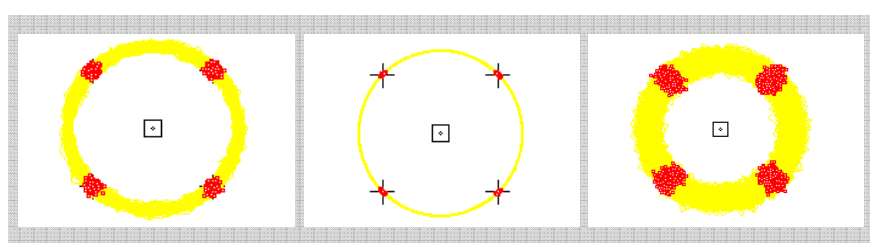

(j)

(k)

(1)

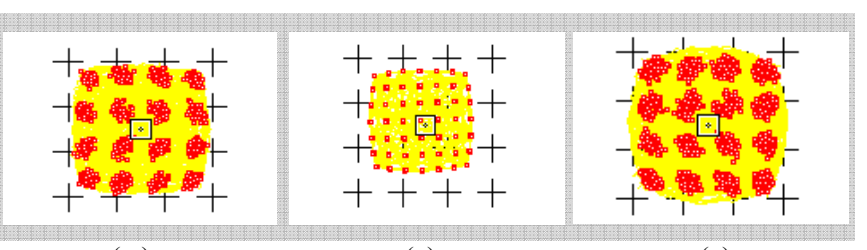

(m)

(n)

(o)

Figure 6. Constellation diagram results: (a) BPSK air channel, $\mathrm{SR}=100 \mathrm{Ksps}$ and DTR $=60 \mathrm{~cm}$, (b) BPSK body channel, SR $=100 \mathrm{Ksps}$ and DTR $=60 \mathrm{~cm}$, (c) BPSK body channel, $\mathrm{SR}=2.5 \mathrm{Msps}$ and $\mathrm{DTR}=155 \mathrm{~cm}$, (d) QPSK air channel, $\mathrm{SR}=100 \mathrm{Ksps}$ and DTR $=60 \mathrm{~cm}$, (e) QPSK body channel, $\mathrm{SR}=100 \mathrm{Ksps}$ and $\mathrm{DTR}=60 \mathrm{~cm}$, (f) QPSK body channel, $\mathrm{SR}=2.5 \mathrm{Msps}$ and $\mathrm{DTR}=155 \mathrm{~cm},(\mathrm{~g})$ $8 \mathrm{PSK}$ air channel, $\mathrm{SR}=100 \mathrm{Ksps}$ and $\mathrm{DTR}=60 \mathrm{~cm}$, (h) $8 \mathrm{PSK}$ body channel, $\mathrm{SR}=100 \mathrm{Ksps}$ and $\mathrm{DTR}=60 \mathrm{~cm}$, (i) $8 \mathrm{PSK}$ body channel, $\mathrm{SR}=2.5 \mathrm{Msps}$ and $\mathrm{DTR}=155 \mathrm{~cm}$, (j) MSK air channel, $\mathrm{SR}=100 \mathrm{Ksps}$ and DTR $=60 \mathrm{~cm},(\mathrm{k}) \mathrm{MSK}$ body channel, $\mathrm{SR}=100 \mathrm{Ksps}$ and $\mathrm{DTR}=60 \mathrm{~cm},(1) \mathrm{MSK}$ body channel, $\mathrm{SR}=2.5 \mathrm{Msps}$ and $\mathrm{DTR}=155 \mathrm{~cm},(\mathrm{~m}) 16 \mathrm{QAM}$ air channel, $\mathrm{SR}=100 \mathrm{Ksps}$ and $\mathrm{DTR}=60 \mathrm{~cm}$, (n) $16 \mathrm{QAM}$ body channel, $\mathrm{SR}=100 \mathrm{Ksps}$ and $\mathrm{DTR}=60 \mathrm{~cm}$, (o) 16QAM body channel, $\mathrm{SR}=2.5 \mathrm{Msps}$ and $\mathrm{DTR}=155 \mathrm{~cm}$.

maximum values considered in this paper yields a very high level of distortion in all modulation schemes and, therefore, a decrease of the quality of the signal transmission. Only BPSK provides received symbols surrounding the reference points, although quite distant due to the high distortion. The received symbols of rest of modulation schemes are dispersed along all the constellation diagrams as it is shown in the case of 8PSK.

\section{2) Eye Diagram}

Eye diagram is also a good performance measure for digital modulations in order to evaluate the quality of the signal transmission. The better quality the digital signal transmission, the more open white space there should be in the eye. Figs. 7a,

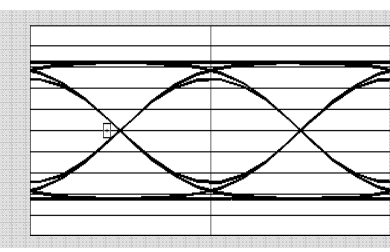

(a)

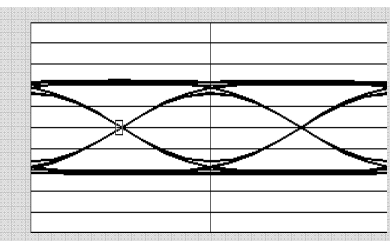

(c)

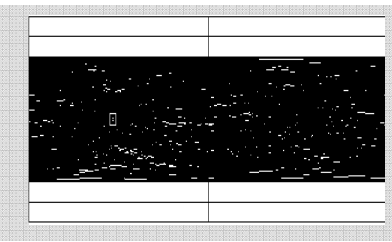

(e)

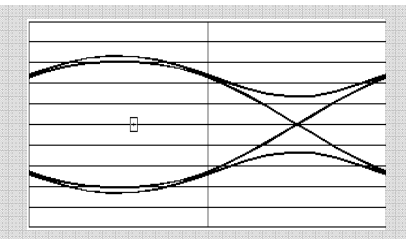

(g)

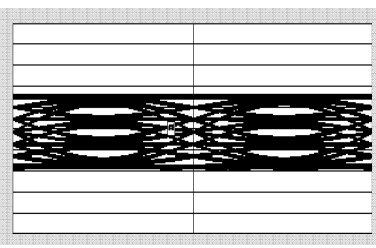

(i)

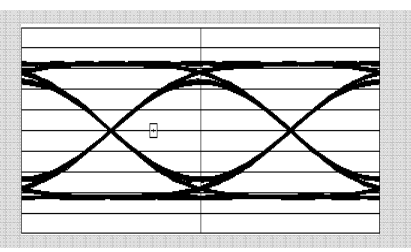

(b)

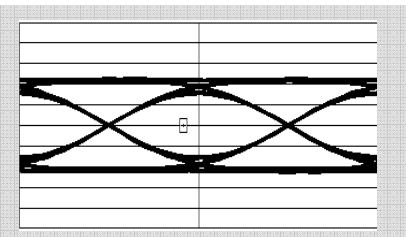

(d)

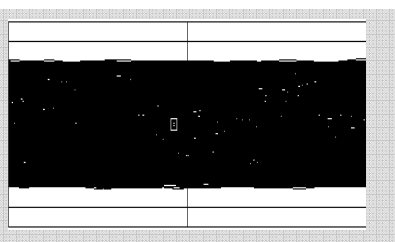

(f)

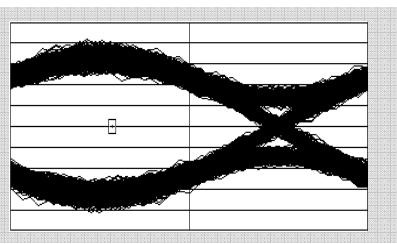

(h)

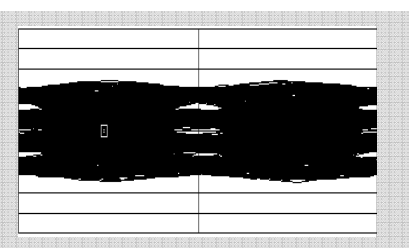

(j)
Figure 7. Eye diagrams. (a) BPSK $\mathrm{SR}=100 \mathrm{~K}$ sps and DTR $=60 \mathrm{~cm}$, (b) BPSK $\mathrm{SR}=2.5 \mathrm{Msps}$ and DTR $=155 \mathrm{~cm}$, (c) QPSK SR=100Ksps and DTR $=60 \mathrm{~cm}$, (d) QPSK SR $=2.5 \mathrm{Msps}$ and $\mathrm{DTR}=155 \mathrm{~cm}$, (e) $8 \mathrm{PSK} \mathrm{SR}=100 \mathrm{Ksps}$ and

$\mathrm{DTR}=60 \mathrm{~cm}$, (f) $8 \mathrm{PSK} \mathrm{SR}=2.5 \mathrm{Msps}$ and $\mathrm{DTR}=155 \mathrm{~cm},(\mathrm{~g}) \mathrm{MSK} \mathrm{SR}=100 \mathrm{Ksps}$ and DTR $=60 \mathrm{~cm}$, (h) MSK SR $=2.5 \mathrm{Msps}$ and DTR $=155 \mathrm{~cm}$, (i) $16 \mathrm{QAM}$

$\mathrm{SR}=100 \mathrm{Ksps}$ and $\mathrm{DTR}=60 \mathrm{~cm}$, (j) $16 \mathrm{QAM} \mathrm{SR}=2.5 \mathrm{Msps}$ and $\mathrm{DTR}=155 \mathrm{~cm}$.

$7 \mathrm{c}, 7 \mathrm{e}, 7 \mathrm{~g}$ and $7 \mathrm{i}$ show the experiment results of the intra-body propagation channel when symbol rate is $100 \mathrm{Ksps}$ and the distance is $60 \mathrm{~cm}$ (same conditions as in Figs. 6b, 6e, 6h, 6k and $6 \mathrm{n}$ ) and Fig. $7 \mathrm{~b}, 7 \mathrm{~d}, 7 \mathrm{f}, 7 \mathrm{~h}$ and $7 \mathrm{j}$ when symbol rate is $2.5 \mathrm{Msps}$ and the distance is $155 \mathrm{~cm}$ (same measurement conditions as in Figs. 6c, 6f, 6i, 61 and 6o). When symbol rate is 100Ksps and the distance is $60 \mathrm{~cm}$, BPSK, QPSK and MSK yield good performance with big white space in the center of the eye diagram (see Fig. 7a, 7c and $7 \mathrm{~g}$ respectively). The eyes of 8PSK and 16QAM are small, which it indicates poor received signal quality. On the other hand, when the symbol rate and the distance between TX and RX are increased, the jitter and the voltage level errors increase and, as a result, the height and width of the eye decrease in all modulation techniques. BPSK (Fig. 7b) and QPSK (Fig. 7d) are the most resistant modulation schemes to the increase of transmission rate and distance, and 
8PSK (Fig. 7f) and 16QAM (Fig. 7j) are the most sensitive techniques. MSK still provides good results although the eye is reduced (see Fig. 7h).

As in the previous subsection, we have observed that, if the distance and the symbol rate are increased to the maximum values, only BPSK provides relatively good performance with white space in the center of the eye, although much smaller than in Fig. $7 \mathrm{~b}$ due to the rise of the error levels.

\section{3) $E V M$}

EVM is an important metric for testing the modulation accuracy and therefore the quality of the communication link. EVM quantifies the difference between the ideal signal and the measured signal. A large EVM degrades transceiver performance by causing poor detection accuracy and, therefore, low modulation quality. Mobile communication standards define EVM requirements for the modulation techniques employed in their systems. GSM, EDGE, 3G WCDMA and IEEE 802.15.4 set up that EVM shall not be worse than 7\% for MSK [10], 9\% for 8PSK [11], 17.5\% for QPSK [12], 12.5\% for 16QAM [12] and 35\% for BPSK [13] respectively. In the measurements, we take these requirements as the thresholds for our evaluation. However, in the case of BPSK, we find that the EVM requirement is too high if we compare the constellation and eye diagram results with the EVM results. Therefore, we decide to decrease the EVM threshold for BPSK to $20 \%$.

Figs. 8, 9, 10, 11 and 12 presents the EVM results of the

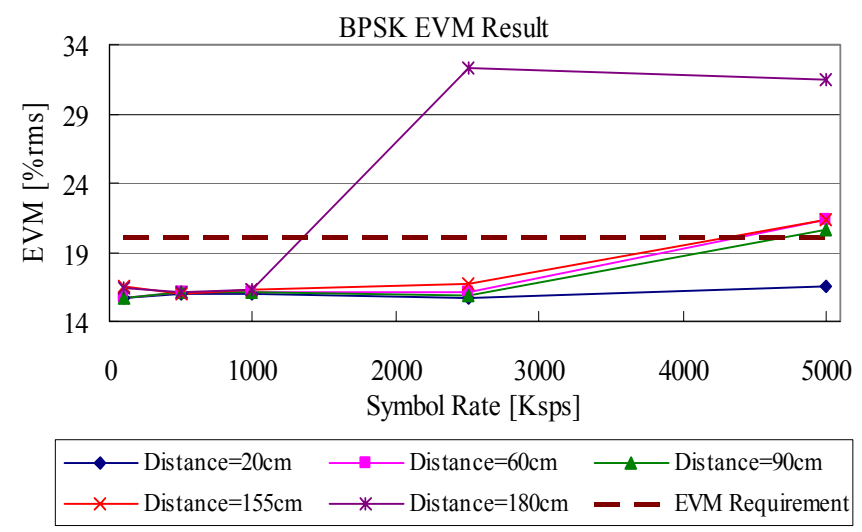

Figure 8. EVM results of BPSK.

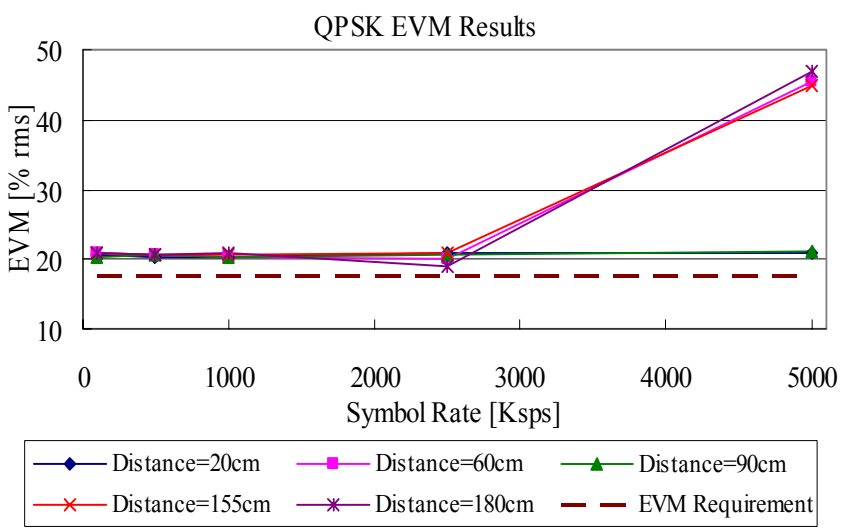

Figure 9. EVM results of QPSK. five modulation schemes in terms of root mean square (RMS) percentage and as a function of the symbol rate. The figures show the results of five different distances between TX and RX (i.e. $20 \mathrm{~cm}$ and $60 \mathrm{~cm}$ from arm-arm model, $90 \mathrm{~cm}$ and $155 \mathrm{~cm}$ from foot-head model and $180 \mathrm{~cm}$ from foot-arm model). Fig. 8 illustrates that BPSK yields lower results than the EVM requirement for symbol rates up to $5 \mathrm{Msps}$ when the shortest distance is considered, symbol rates up to $2.5 \mathrm{Msps}$ when distance is from $60 \mathrm{~cm}$ to $155 \mathrm{~cm}$ and symbol rates up to $1 \mathrm{Msps}$ when the largest distance. Fig. 9, 10 and 12 show respectively that the EVM results of QPSK, 8PSK and 16QAM are worse than the EVM requirements for all the symbol rates and distances considered. Finally, Fig. 11 shows that MSK yields good results with EVM measurements below the EVM requirement for symbol rates up to $5 \mathrm{Msps}$ when distance is $20 \mathrm{~cm}$, symbol rates up to $2.5 \mathrm{Msps}$ when distance is $60 \mathrm{~cm}$ and $90 \mathrm{~cm}$, symbol rates up to $1 \mathrm{Msps}$ when distance is $155 \mathrm{~cm}$ and symbol rates up to $500 \mathrm{Ksps}$ when the largest distance is considered.

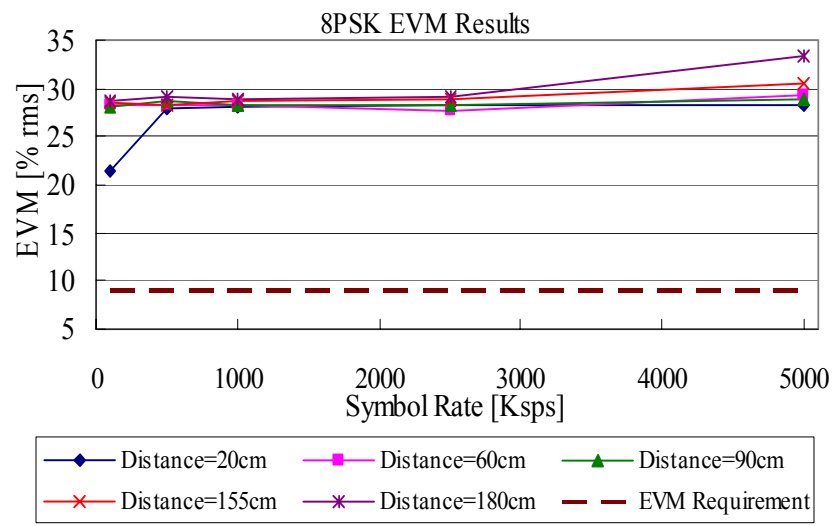

Figure 10. EVM results of 8PSK.

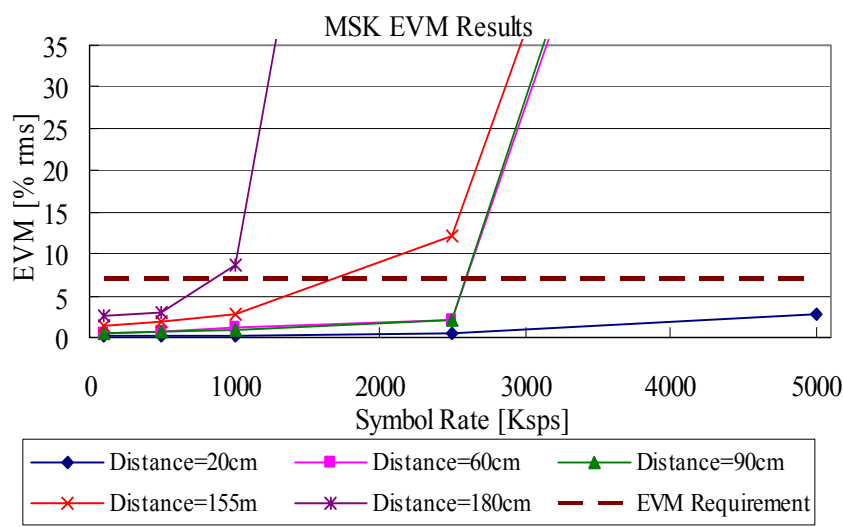

Figure 11. EVM results of MSK. 


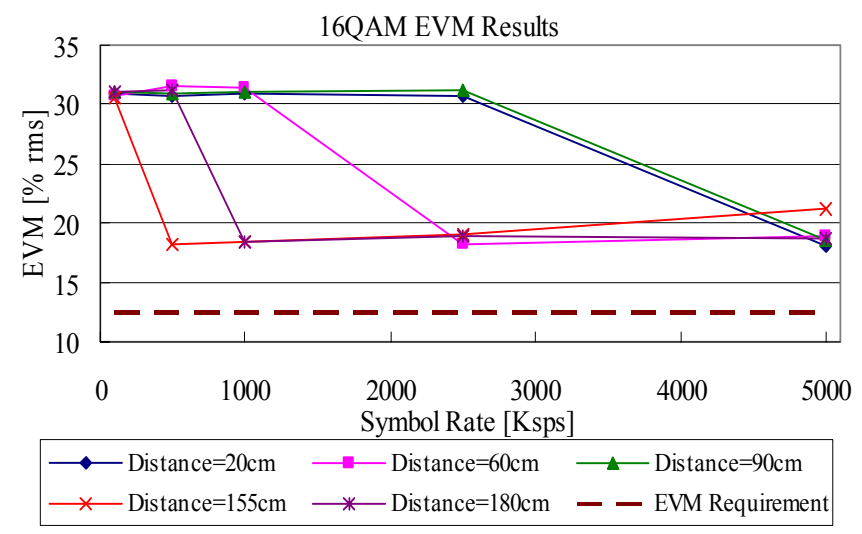

Figure 12. EVM results of 16QAM.

\section{CONCLUSIONS}

In this paper, we have investigated the transmission characteristics of the human body considering high radio frequency signals. The experiment results of the frequency domain response have shown that the received signal power decreases when the frequency increases, and the power decrease is faster when the distance between TX and RX is bigger. From the results, we believe that the frequency range up to $600 \mathrm{MHz}$ could be suitable for IBC.

On the other hand, we have evaluated five modulation schemes for IBC in terms of constellation diagram, eye diagram and EVM. Experiment results have shown that MSK and BPSK are the most suitable modulation schemes for IBC with the EVM results lower than the EVM requirements, close received symbols to the constellation points and big white space in the center of the eye diagram. Moreover, the results have proved that both MSK and BPSK can achieve high transmission rates. However, we have observed that increasing the distance between TX and RX and the symbol rate causes a rise of the distortion and, therefore, a decrease of the digital signal quality. As a consequence, MSK and BPSK provide good performance for symbol rates up to $5 \mathrm{Msps}$ in case of the shortest distance between TX and RX considered in this paper (i.e. $20 \mathrm{~cm}$ ), but only for symbol rates up to $500 \mathrm{Ksps}$ (in MSK) and $1 \mathrm{Msps}$ (in BPSK) in case of the largest distance (i.e. $180 \mathrm{~cm})$.

\section{REFERENCES}

[1] Wireless World Research Forum, "Book of Visions 2001," December 2001, www.wwrf.org

[2] J. Agud Ruiz, S. Shimamoto, "Novel Communication Services Based on Human Body and Environment Interaction: Applications inside Trains and Applications for Handicapped People", IEEE Wireless Communications and Networking Conference 2006, Las Vegas, USA, April 2006.

[3] T. G. Zimmerman, "Personal Area Networks: Near-filed Intra-Body Communication,” IBM Systems Journal, Vol .35, N. 3\&4, pp. 609-617, 1996.

[4] Matsushita Electric Works Ltd., 2004, www.mew.co.jp/epress/2004/0409-02.htm

[5] M. Shinagawa et al., "A Near-Field-Sensing Transceiver for Intrabody Communication Based on the Electrooptic Effect," IEEE Transactions on instrumentation and measurement, Vol. 53, NO. 6, December 2004.

[6] K. Hachisuka Post et al., "Development and Performance Analysis of an Intra-Body Communication Device," Proc. of 12th International Conference on Solid State Sensors, Actuators and Microsystems, Boston, pp. 1722-1725, June 2003.

[7] K.Fujii, K. Ito, "Evaluation of the Received Signal Level in Relation to the Size and Carrier Frequencies of the Wearable Device Using Human Body as a Transmission Channel," Proc. of 2004 Antennas and Propagation Society Symposium, pp. 105-108, June 2004.

[8] J. Agud Ruiz, S. Shimamoto, "A Study on the Transmission Characteristics of the Human Body Towards Broadband Intra-body Communications", IEEE 9th International Symposium on Consumer Electronics 2005, Macau, China, 14-16 June 2005.

[9] World Health Organization, "Electromagnetic Fields $(300 \mathrm{~Hz}$ to 300GHz)," 1993, www.inchem.org/documents/ehc/ehc/ehc137.htm

[10] ETSI GSM 5.05 Standard v.8.4.1: "Digital Cellular Telecommunications System (phase 2+), Radio Transmission and Reception", 1999

[11] P. Morgan, "Radio Design Considerations for EDGE Handsets", Silicon Laboratories Inc., www.silabs.com

[12] 3GPP TS 25.101 v.6.8.0: "User Equipment (UE) radio transmission and reception (FDD)", June 2005

[13] K. T. Le, "Designing a ZigBee-ready IEEE 802.15.4-compliant radio transceiver", www.rfdesign.com, November 2004 